\title{
Oral hypoglycaemic activity of a commercially available herbal preparation.
}

\author{
M. C. P. Canagaratna' and A. V. M. Sivagowry' \\ The Ceylon Journal of Medical Science 1998; 41: 25-30
}

\section{Summary}

The aim of this study was to establish the oral hypoglycaemic activity of a commercially available herbal powder (mixture of equal parts of Salacia reticulata Wight, Strychnos potatonum, L.F. Cardiospermum microcarpum, H. B. K. and Santalum album, L) used in the treatment of diabetes mellitus.

This was shown by.demonstrating the ability of the herbal mixture given orally, at a dosage of $2 \mathrm{mg} / 100 \mathrm{~g}$ body weight, to lower the fasting blood glucose levels and to improve glucose tolerance in healthy male Sprague - Dawley rats.

The results indicate that the extract significantly lowered the fasting blood glucose levels in the treatment group 1 h., 2 h., and $3 h$. after oral administration of the extract, when compared with those of the control group which received distilled water ( $p<0.05,<0.01,<0.01$ respectively). Maximum hypoglycaemic activity $(12.2 \%$ reduction of fasting blood glucose) was observed 2 hours after administration of the extract. When the glucose tolerance test was performed, the control group of animals given distilled water, followed one hour later by an oral dose of $50 \%$ $w / v$ glucose solution, showed the maximum increase $(51.1 \%)$ in blood glucose concentration after one hour. In contrast, the animals treated with the extract showed a- significant improvement in their ability to utilise an external glucose load (maximum increase was only $28.8 \%$ ) $(p<0.001)$. The area under the curve of percentage increase in blood glucose was also significantly less in the treatment group than in the control group ( $p<0.001)$.

\section{Introduction}

Despite the progress of conventional chemistry and pharmacology in producing effective drugs, several thousands of medicinal plants are still used throughout the world in therapeutics, including the treatment of diabetes mellitus. The aqueous extracts of plants are orally administered to control diabetes mellitus $(1,2,3)$. Different parts such as bark, roots, leaves and stems either singly or in combination are prescribed by Ayurvedic physicians in the treatment of diabetes. Though more than 400 such plants are reputed to have hypoglycaemic activity, only a few of them have received adequate medical or scientific evaluation (4).

In Sri Lanka, approximately 40 plants are reputed to have oral hypoglycaemic activity (1). Among these, the most widely used are Momordica charantia, L, Ficus benghalensis, L, Aegle marmelos, (L.) Corr, Salacia reticulata, Wight and Gymnema sylvestre, R. Br. ex Schult.

The herbal mixture with the claimed oral hypoglycaemic activity, used in this study consists of S. reticulata (family Hippocrateaceae; Sinhala - Kothalahimbutu), Strychnos potatorum. L.F. (family - Loganiaceae; Sinhala - Ingini), Cardiospermum microcarpum H.B.K. (family Sapindaceae; Sinhala - Welpenala) and Santalum album L. (family-Santalaceae; Sinhala - Sudu Handun) (5). Though the aqueous extract of $S$. reticulata root bark has been shown to possess oral hypoglycaemic activity (6) no precious study has been done to determine the hypoglycaemic activity of the particular combination of herbs in the mixture under investigation.

1: Associnte Professor, Department of Biochemistry and Molecular Biology, Faculty of Medicine, University of Colombo, Kynsey Road, Colombo.

2. Research Student, Department of Biochemistry and Molecular Biology, Faculty of Medicine, University of Colombo, Kynsey Rond, Colombo. 
The present study was undertaken because of the widespread use of the herbal mixture by diabetics in Sri Lanka. Its popularity is evidenced by the production of the preparation in commercial quantities by a company in Colombo. Hence there is both a need as well as a justification for a close scrutiny of the reported efficacy of this preparation in the treatment of diabetes mellitus.

\section{Materials and Methods}

All experiments were carried out at room temperature $\left(30^{\circ} \mathrm{C} \pm 3^{\circ} \mathrm{C}\right)$.

\section{Experimental animals}

Healthy adult male Sprague - Dawley rats (body weight $200 \mathrm{~g} \pm 50 \mathrm{~g}$ ) were maintained on a standard laboratory diet purchased from the Oils and Fats Corporation of Sri Lanka. The animals were fasted overnight (16-18 hours) before the commencement of all experiments.

\section{Preparation of the herbal extract}

Each of the herbs in the mixture was purchased in a powdered form from William Grinding Mills, Dehiwela. The products did not carry an expiry date and were tested within two weeks of purchase. The herbal powders were mixed in equal quantities and an extract was prepared as follows: $10 \mathrm{~g}$ of the mixture was boiled with 1000 $\mathrm{mL}$ of distilled water for about $1 \mathrm{~h}$ until the final volume was $500 \mathrm{~mL}$. This was centrifuged for 5 min. at $3000 \mathrm{rpm}$ and the supernatant was used: The method of preparation recommended by the manufacturers is to add $1000 \mathrm{~mL}$ of boiling water to $20 \mathrm{~g}$ of the herbal mixture and keep for 10 minutes. After this period the mixture was centrifuged at $3000 \mathrm{rpm}$ for $5 \mathrm{~min}$. and the supernatant used. As the supernatant prepared by this method did not have any effect on the fasting blood glucose levels, the modified method described above was used.

\section{Administration and dosage of herbal extract}

The drug was administered by oral intubation while the animals were under light diethyl ether anaesthesia. The dosage $(20 \mathrm{mg} / \mathrm{mL} / 100 \mathrm{~g}$ body weight) corresponded to the normal therapeutic dose ( 2 tea spoons $=10 \mathrm{~g}$ ) of the plant extract administered to human adults.

\section{Blood glucose estimation}

Blood samples $(50 \mu \mathrm{L})$ were obtained using a microcap (Drummond Scientific company, U.S.A.) by tail puncture when the animals were under light anaesthesia, and blood glucose was determined by the glucose oxidase method (7).

\section{Effect of the herbal extract on fasting blood glucose level}

Rats were fasted overnight ( $n=28$ ). The animals were randomly divided into two groups after taking blood samples for the determination of fasting blood glucose levels. The control group received distilled water $(1 \mathrm{~mL} / 100 \mathrm{~g}$ body wt.), while the treatment group received the herbal extract. After the administration. blood samples were collected at $1 \mathrm{~h}$ intervals for $3 \mathrm{~h}$ and the glucose levels estimated.

\section{Effect of the extract on the glucose tolerance test}

Rats were fasted overnight ( $n=26)$. After taking blood samples for the determination of fasting blood glucose levels, they were divided into two groups. The control group received distilled water, while the treatment group received the herbal extract. One hour after the administration, all received an oral dose $(1 \mathrm{~mL} / 100 \mathrm{~g}$ body $w \mathrm{t}$.) of glucose solution $(50 \% \mathrm{w} / \mathrm{v})$. Blood samples were collected at $1 \mathrm{~h}$ intervals for $3 \mathrm{~h}$ and glucose levels estimated. The area under the curve of percentage increase of blood glucose concentrations for each animal was calculated as follows: (8)

first + last concentrations

Total area $=$ time interval $x-\frac{}{\cdot}+\begin{gathered}\text { sum of all other } \\ \text { concentrations }\end{gathered}$

\section{Results and Discussion}

The effect of the herbal extract on fasting blood glucose is given in Figure 1. It is clear that in the treatment group, there was a decrease in the fasting blood glucose values expressed as a percentage of the zero time blood glucose values at 1, 2 and $3 \mathrm{~h}$ after the administration of the extract. The maximum effect $(12.2 \%$ reduction, $\mathrm{p}<0.001$ ) was seen at $2 \mathrm{~h}$. In contrast, the corresponding curve for the control group showed little deviation from the zero time level. 


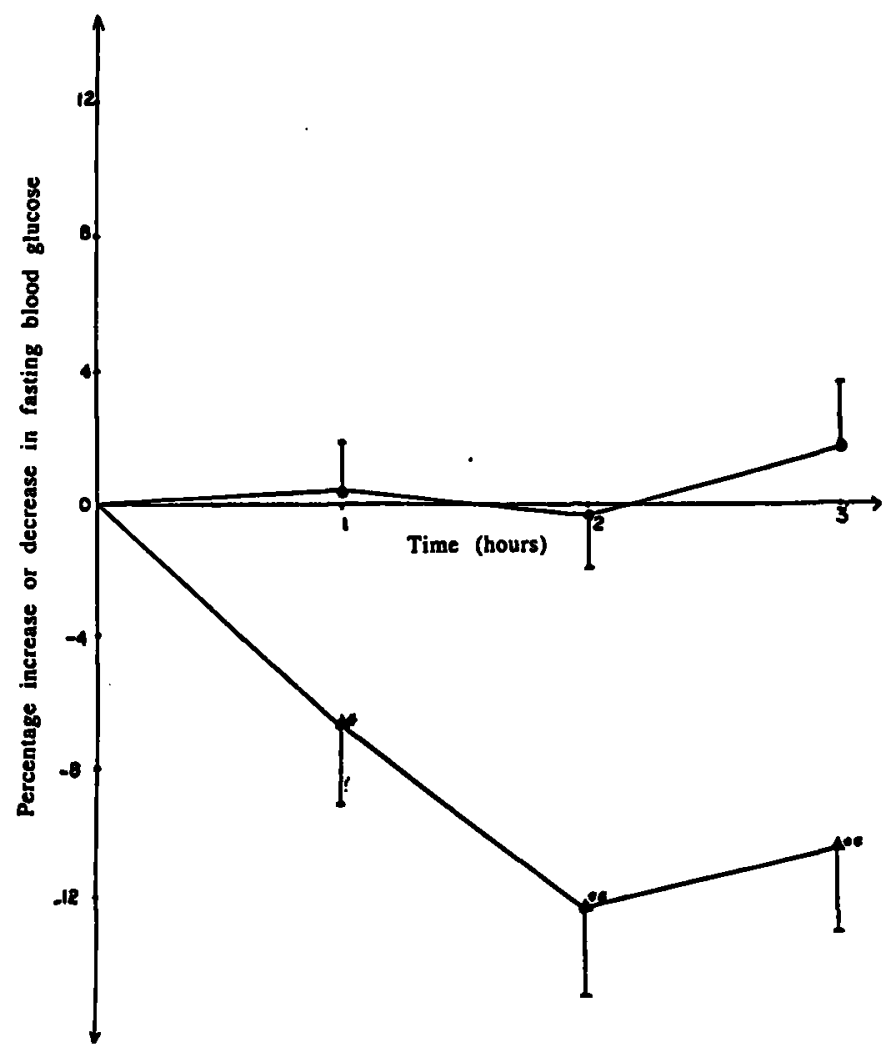

Figure 1

Effect of the herbal mixture on fasting blood glucose levels. Two groups of fasted rats $(n=28)$ were orally administered either the extract of the herbal mixture or distilled water $(1 \mathrm{~mL} / 100 \mathrm{~g}$ body wt.) Blood samples $(50 \mu \mathrm{L})$ were collected at zero time and at $1 \mathrm{~h}$ intervals for glucose assay. Results are expressed as percentage increase/decrease of blood glucose with respect to the zero time blood glucose values. Results of 3 experiments were pooled. Mean \pm SEM values are given.

- distilled water. $\sim \mathbf{\Delta}$ herbal mixture.

"p $<0.05 \quad$ ** $p<0.01$

The effect of the herbal extract on oral glucose tolerance is illustrated in Figure 2. The results are given as a percentage increase of the zero time fasting blood glucose level. The control group that received distilled water, followed $1 \mathrm{~h}$. later by an oral dose of glucose, showed the maximum increase $(51.1 \%)$ in blood glucose concentration after $1 \mathrm{~h}$. In contrast, the animals treated with the herbal extract showed a significant improvement in their ability to utilize an external glucose load.
The maximum increase in this group, also after $1 \mathrm{~h}$ was only $28.8 \%$ ( $p<0.001)$. The area under the curve of percentage increase in blood glucose after administration of the oral glucose load was also significantly less in the treatment group (55.9) than in the control group (105.8) $(\mathrm{p}<0.001)$ [see Fig. 3].

Hence, the orally administered herbal extract significantly reduced not only the fasting blood 
glucose level in healthy rats but also significantly improved their tolerance of an external glucose load.

The root bark of $S$. reticulata has been shown by Karunanayake et al(6) to have hypoglycaemic activity when administered orally to healthy laberatory rats. In the abovestudy, $250 \mathrm{~g}$ of dried powdered root bark of the plant was boiled in $1000 \mathrm{~mL}$ distilled water for 3 hours and the final volume was reduced to $100 \mathrm{~mL}$ in vacuo. This $250 / \mathrm{dL}$ extract caused a $30 \%$ reduction in fasting blood glucose levels at a dosage of 1 $\mathrm{mL} / 100 \mathrm{~g}$ body wt. The concentrations used in the above study (6) are much higher than those used by us.

In the present study, a $2 \mathrm{~g} / \mathrm{dL}$ extract of the herbal mixture produced a $12.2 \%$ reduction in fasting blood glucose levels.Therefore, $40 \%$ of the hypoglycaemic effect of S. reticulata shown in the study of Karunanayake et al. was shown in this study by an extract of a mixture almost 125 times less concentrated.

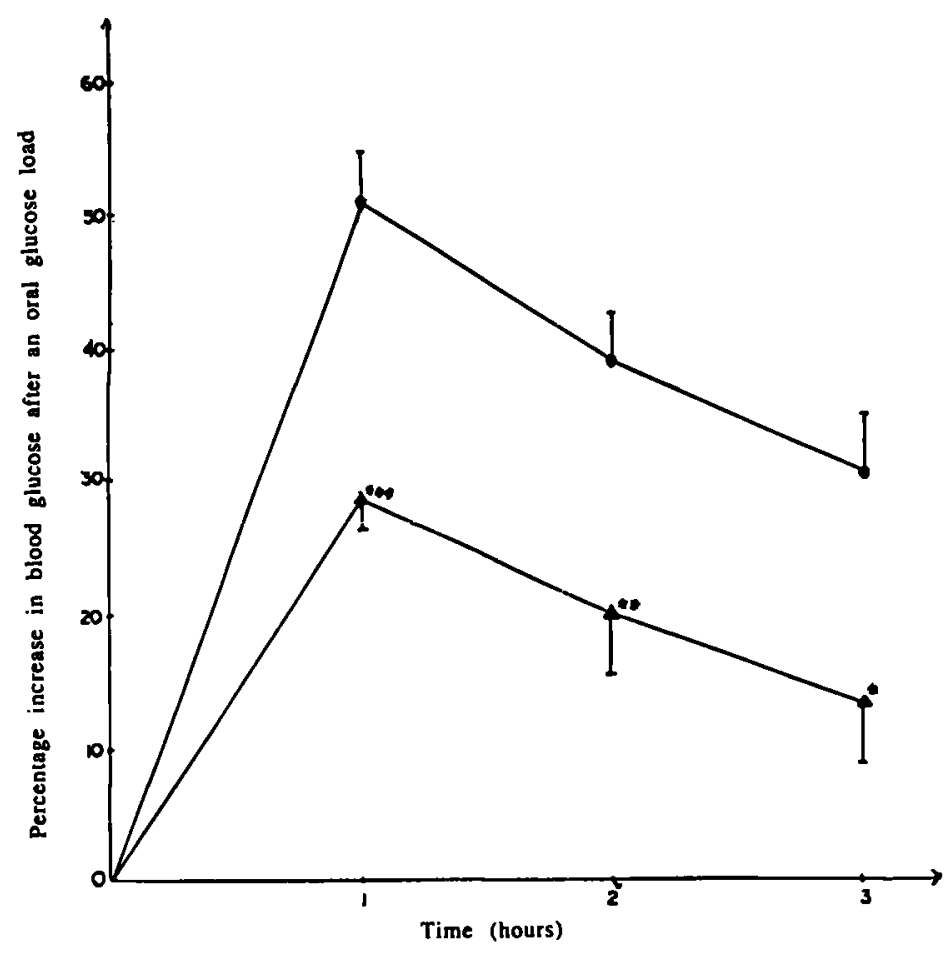

Figure 2

Effect of the herbal preparation on the oral glucose tolerance of rats. Fasted rats $(n=26)$ were administered distilled water/herbal mixture $(1 \mathrm{~mL} / 100 \mathrm{~g}$ body $\mathrm{wt}$.) $1 \mathrm{~h}$ prior to an oral glucose load (1 $\mathrm{mL}$ of $50 \% \mathrm{w} / \mathrm{v}$ solution $/ 100 \mathrm{~g}$ body $\mathrm{wt}$.). Blood samples $(50 \mu \mathrm{L})$ were collected at zero time and at $1 \mathrm{~h}, 2$ $\mathrm{h}$ and $3 \mathrm{~h}$ after the glucose load for assay of blood glucose. Results are expressed as percentage increase of blood glucose with respect to fasting blood glucose values. Results of 3 experiments were pooled. Mean \pm SEM values are given.

$\longrightarrow$ distilled water. $\longrightarrow$ herbal mixture.

${ }^{*} \mathrm{p}<0.02 \quad{ }^{* *} \mathrm{p}<0.01 \quad{ }^{* * *} \mathrm{p}<0.001$ 
It is likely that more than one of the herbs contributed to the hypoglycaemic activity. Further work should therefore be directed towards investigating the hypoglycaemic activity of each of the four herbs singly and in various combinations to identify those herbs in the preparation contributing to the hypoglycaemic activity and those which may have a potentiating effect or which, possibly, are included to counteract any toxic effects.

Further, evidence of chronic toxic effects should also be sought. Evidence of hepatic toxicity, if

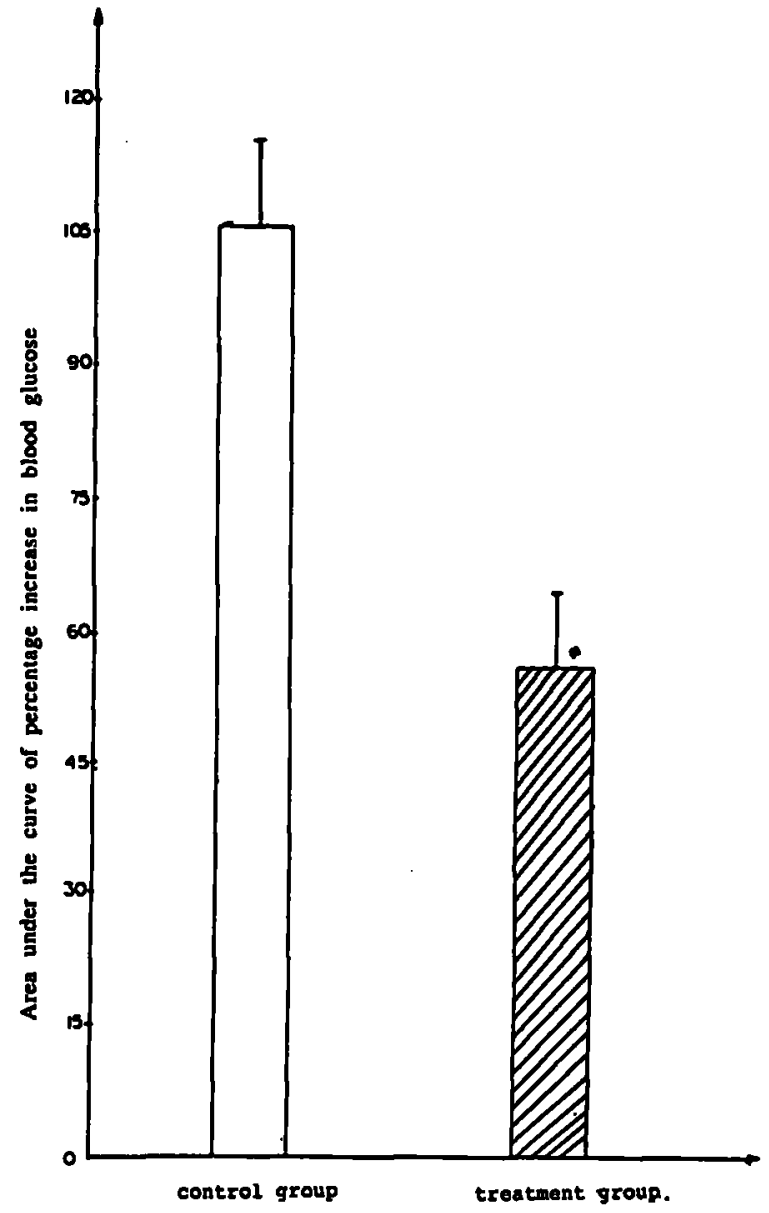

Figure 3 any, can be studied by the assay of serum levels of hepatic enzymes which include alkaline phosphatase, gamma-glutamyl transpepatidase and the aminotransferases and by histopathological studies. Renal toxicity, if any, can be investigated by assay of $\mathrm{N}$-acetyl $\beta$ - $\mathrm{D}$ glucosaminidase and by histopathological studies.

In addition, the effect of this preparation on rats rendered diabetic by streptozotocin or alloxan treatment should be studied before studies on diabetic patients are undertaken.

Mean and S.E.M. of areas under the curves of percentage increase of blood glucose concentrations, shown in Fig 2. The bar charts represent the glucose tolerance curve areas of animals treated with distilled water and with herbal mixture.

$\square$-control group -test group ${ }^{*} p<0.001$. 


\section{Acknowledgements}

This work was done in part fulfillment for the M.Sc. degree and was supported by the Department of Biochemistry and Molecular Biology, Faculty of Medicine, University of Colombo.

\section{References}

1. Attygalle J. Sinhalese Materia Medica: 1917 Apothecaries Press, Colombo.

2. Bever BO, Zahnd GR, Plants with oral hypoglycaemic action. Quarterly Journal of Crude Drug Research 1979; 17: 139-196.

3. Farnsworth NR., Segelman AB. Hypoglycaemic Plants. Tile \& Till 1971; 57: 52-55.

4. Bailey C J., Day C. Traditional plant medicines as treatments for diabetes. Diabetes Care 1989; 12: 553-564.
5. Jayaweera DMA. Medicinal plants used in Ceylon. Colombo: National Science Council of Sri Lanka. 1981; 77, 1982(3), 277, 1982(5), 43,49 .

6. Karunanayake EH., Welihinda J. Sirimanne SR., Sinnadurai G. Oral hypoglycaemic activity of some medicinal plants of Sri Lanka. Journal of Ethnopharmacology 1984; 11: 223-231.

7. Hugget A. St G, Nixon DA. Use of glucose oxidase, peroxidase \& O-dianisidine in determination of blood and urinary glucose. Lancet 1957; iii: 368-370.

8. Rowland M, Tozer TN. Assessment of area. Clinical pharmacokinetics: Concepts \& Applications. 2nd Ed. London. Lea \& Febiger (U.K.) Ltd. 1989; 459-463. 